\title{
MAGNETIC GROUND STATE OF FULLERENES
}

\author{
A. Koper ${ }^{a}$, J. Stankowski ${ }^{b}$ AND M. Thomas ${ }^{a}$ \\ aSolid State Theory Division, Institute of Physics, A. Mickiewicz University \\ Matejki 48/49, 60-769 Poznań, Poland \\ ${ }^{b}$ Institute of Molecular Physics, Polish Academy of Sciences \\ Smoluchowskiego 17/19, 60-179 Poznań, Poland
}

The classical limit of the effective antiferromagnetic Heisenberg Hamiltonian has been investigated on the series of free clusters (molecules) of $\mathrm{C}_{N}$ ( $N=32,44,50,58,60,70$ and 84$)$. The valley structures of the frustrated ground states were determined. Moreover, the probability distributions of the spin correlation functions were calculated.

PACS numbers: 75.10.Hk, 75.25.+z, 36.40.+d

It was found [1] that in the classical approximation the magnetic ground state of some fullerenes is exotic with nontrivial topology. It is caused by frustrations of interspins interactions due to the presence of pentagons in the specific spherical geometry of $\mathrm{C}_{N}$ clusters.

In the present paper we want to analyse in detail magnetic ground states of the whole series of $\mathrm{C}_{N}$ clusters. Moreover, it is interesting to find out some differences among the ground state properties of respective clusters, and then to verify if the $\mathrm{C}_{60}$ one is an unique fullerene as suggested in [1]. In order to obtain magnetic ground states of the clusters in the semi-classical approach we use a variational method. The employed Monte Carlo relaxation procedure is based on the idea of a fast cooling and seems to be most relevant to phenomena observed empirically (cf. [2]).

We investigate the series of free $\mathrm{C}_{N}$ clusters employing the effective antiferromagnetic Heisenberg Hamiltonian

$$
H=-\frac{1}{2} \sum_{i, j} J_{i j} \widehat{S}_{i} \cdot \widehat{S}_{j} .
$$

The Hamiltonian arises from the Hubbard one describing the singly-occupied $\pi$-orbitals at the carbon sites assuming the strong enough on-site Coulomb repulsion (cf. [1]).

Our approach is a slightly modified Ritz variational method. We start with a subset $\Omega$ of trial functions $\varphi$ of the Hilbert space $\mathcal{H}$ of the Hamiltonian $H$. Then we look for a map $F: \Omega \rightarrow \Omega$ so as to its successive iterations $\varphi_{n}=F_{n}(\varphi)$, $F_{n}=F(F(\ldots F) \ldots)$, minimize the functional $R(\varphi)=\langle\varphi|H| \varphi\rangle /\langle\varphi \mid \varphi\rangle$ for every $\varphi \in \Omega$. The stable points of $F$, i.e. the solutions of the equation $\varphi=F(\varphi)$, are the requested local minima in $\Omega$. 
Within the presented framework we specify the mapping $F$ on the set $\Omega$ of the coherent spin states $|s\rangle$ of the spin operator $\widehat{S}: \widehat{S}|s\rangle=S s|s\rangle$. $s$ are the unit vectors. Moreover, $\langle s \mid s\rangle=s \cdot s=1$. Thus in the case the proposed description of fullerenes is essentially equivalent to the consideration of the classical limit of the Hamiltonian (1). A given cluster $\mathrm{C}_{N}$ is now described by a system of $N$ spins $\vec{s}=\left(s_{1}, s_{2}, \ldots, s_{N}\right)$ and the appropriate coherent state is a tensor product $|\vec{s}\rangle=\left|s_{1}\right\rangle\left|s_{2}\right\rangle \ldots\left|s_{N}\right\rangle$. Then $R(|\vec{s}\rangle)=-\frac{1}{2} \sum_{i, j} \tilde{J}_{i j} s_{i} \cdot s_{j}$, where $\tilde{J}_{i j}=J_{i j} S_{i} S_{j}$.

Finally, the procedure gives rise to the set of equations

$$
\boldsymbol{s}_{i}=F_{i}(\vec{s})=\sum_{j} \tilde{J}_{i j} \boldsymbol{s}_{j} /\left\|\sum_{j} \tilde{J}_{i j} \boldsymbol{s}_{j}\right\|
$$

$i=1,2, \ldots, N$, where $\|u\|$ is the length of a vector $u$. As well known [3, 4], the iterations $F_{n}(\vec{s})$ are always (for any $\vec{s}$ ) convergent to stable points $\vec{s}_{O \nu}$ of the mapping $F$. The mapping rotates the vectors $\vec{s}$ readjusting them to a direction of local molecular field $\sum_{j} \tilde{J}_{i j} s_{j}$ on every site $i$. Therefore $R\left(\left|F_{n+1}(\vec{s})\right\rangle\right) \leq R\left(\left|F_{n}(\vec{s})\right\rangle\right)$ and $\vec{s}_{o \nu}$ are the local minima of the functional $R$.

We assumed (as the first approximation) that the parameters $J_{i j}=-|J|$ for the nearest-neighbour sites, otherwise being equal to zero. In the first step of the used numerical procedure we randomly chose uniformly in the space $\Omega$ a considerable number $\mathcal{N}\left(\mathcal{N}=10^{4}\right.$ in the presented calculations) of points $\vec{s}_{l}^{\prime}(l=1, \ldots, \mathcal{N})$. Then for every $\vec{s}_{l}^{\prime}$ we found $\vec{s}_{0 l}=\lim _{n \rightarrow \infty} F_{n}\left(\vec{s}_{l}^{\prime}\right)$, i.e. the iteration was stopped as differences between the successive values of the functional $R$ reached an assumed accuracy (eg. $10^{-8}$ ). Thus obtained $\vec{s}_{0 l}$ are all local minima of the functional $R$. The procedure does reflect what is expected to happen in an experiment when a sample being at first at high enough temperature is then rapidly cooled down to near-zero temperature. The low-energy metastable spin configurations $\vec{s}_{0 l}$ reached in this way can be regarded as the ground state, for all practical purposes [2]!

The above described method is different than that used in [1]. In the latter the authors found ground state spin configurations of only last three clusters of the considered series by a simple numerical minimizing the energy over the spin variables. Due to technical reasons (eg. [3]) such a simple procedure was not able to reveal all possible local minima of the energy.

We found that the ground states of the $\mathrm{C}_{N}$ clusters consist even of two $\left(\mathrm{C}_{32}\right.$, $\left.\mathrm{C}_{58}, \mathrm{C}_{60}\right)$ or three $\left(\mathrm{C}_{44}, \mathrm{C}_{70}, \mathrm{C}_{84}\right)$ spin configuration space valleys corresponding to slightly different energy values $E_{i}$ (Table I) and separated by energy barriers. Moreover, some of them disclose nontrivial degeneracy, i.e. several nonequivalent spin configurations correspond to the given energy, and, as indicated in Table II, the net magnetic moments, $M=\left\|\sum_{i=1}^{N} s_{i}\right\|$, computed in states of every valley are not always equal to zero.

As we have mentioned yet, the applied relaxation procedure is a reasonable characterization of the effective freezing seen in a very fast experiment. The cooling process of a $\mathrm{C}_{N}$ cluster is equivalent to a relaxation of its spins to local equilibrium positions. Due to the estimation of the relative frequencies of configurations $\vec{s}_{0 l}$ appearances we could obtain the probabilities $p_{\nu}$ of finding the system in the given spin energy valley (Table III). Thus the method enabled us also to determine probabilities of finding clusters in a possible metastable ground state spin configuration in a powder sample of given molecules $\mathrm{C}_{N}$ after its rapid cooling. 
TABLE I

Local energy minima (the valley structure of the ground states) of the series of fullerenes in the effective coupling $|J|$ units per C-atom. $\left({ }^{*}\right)$ indicates the degeneracy of the energy valley.

\begin{tabular}{l|c|c|c}
\hline \hline & $E_{0}$ & $E_{1}$ & $E_{2}$ \\
\hline $\mathrm{C}_{32}$ & -1.23449 & -1.21619 & - \\
$\mathrm{C}_{44}$ & -1.27508 & $-1.26809^{*}$ & -1.26447 \\
$\mathrm{C}_{50}$ & -1.31064 & - & - \\
$\mathrm{C}_{58}$ & $-1.30863^{*}$ & $-1.30730^{*}$ & - \\
$\mathrm{C}_{60}$ & -1.30902 & -1.30091 & - \\
$\mathrm{C}_{70}$ & -1.33520 & $-1.32777^{*}$ & -1.32773 \\
$\mathrm{C}_{84}$ & -1.36358 & -1.34959 & -1.33981
\end{tabular}

TABLE II

Net magnetic moment in the ground states of the respective fullerenes.

\begin{tabular}{c|c|c|c}
\hline \hline & $M_{0}$ & $M_{1}$ & $M_{2}$ \\
\hline $\mathrm{C}_{32}$ & 0 & 0.0458 & - \\
$\mathrm{C}_{44}$ & 0 & 0.1001 & 0 \\
$\mathrm{C}_{50}$ & 0.2708 & - & - \\
$\mathrm{C}_{58}$ & 0.2380 & 0.2105 & - \\
$\mathrm{C}_{60}$ & 0 & 0 & - \\
$\mathrm{C}_{70}$ & 0 & 0.0099 & 0 \\
$\mathrm{C}_{84}$ & 0 & 0 & 0
\end{tabular}

TABLE III Probabilities of finding the fullerenes in their respective energy valleys.

\begin{tabular}{l|l|c|c}
\hline \hline & \multicolumn{1}{|c|}{$p_{0}$} & $p_{1}$ & $p_{2}$ \\
\hline $\mathrm{C}_{32}$ & 0.977 & 0.023 & - \\
$\mathrm{C}_{44}$ & 0.4614 & 0.5382 & 0.0004 \\
$\mathrm{C}_{50}$ & 1 & - & - \\
$\mathrm{C}_{58}$ & 0.8758 & 0.1242 & - \\
$\mathrm{C}_{60}$ & 0.5668 & 0.4332 & - \\
$\mathrm{C}_{70}$ & 0.6752 & 0.3244 & 0.0004 \\
$\mathrm{C}_{84}$ & 0.9194 & 0.0586 & 0.022
\end{tabular}


Moreover, within the approach, we are able to calculate a probability distribution (density) $\langle\delta(x-A)\rangle$ of a physical quantity $A$ in the ground state $(\delta(x)$ is the Dirac $\delta$-function). Namely, the distribution can be obtained after the differentiation of appropriate $\theta$-Heaviside functions

$$
\left.\langle\theta(x-A)\rangle \approx \frac{1}{\mathcal{N}} \sum_{l} \theta(x-A)\left(\vec{s}_{0 l}\right)\right) .
$$

As the example we considered the angle operator

$$
\widehat{\varphi}_{k l}=\arccos \left(S^{-2} \widehat{S}_{k} \cdot \widehat{S}_{l}\right)
$$

between electron spins of $k$ and $l$ sites. The Monte Carlo calculations of the probability distribution of the correlations $\widehat{\varphi}_{k l}$ with respect to a nearest-neighbour pair of spins on pentagons are displayed in Fig. 1.

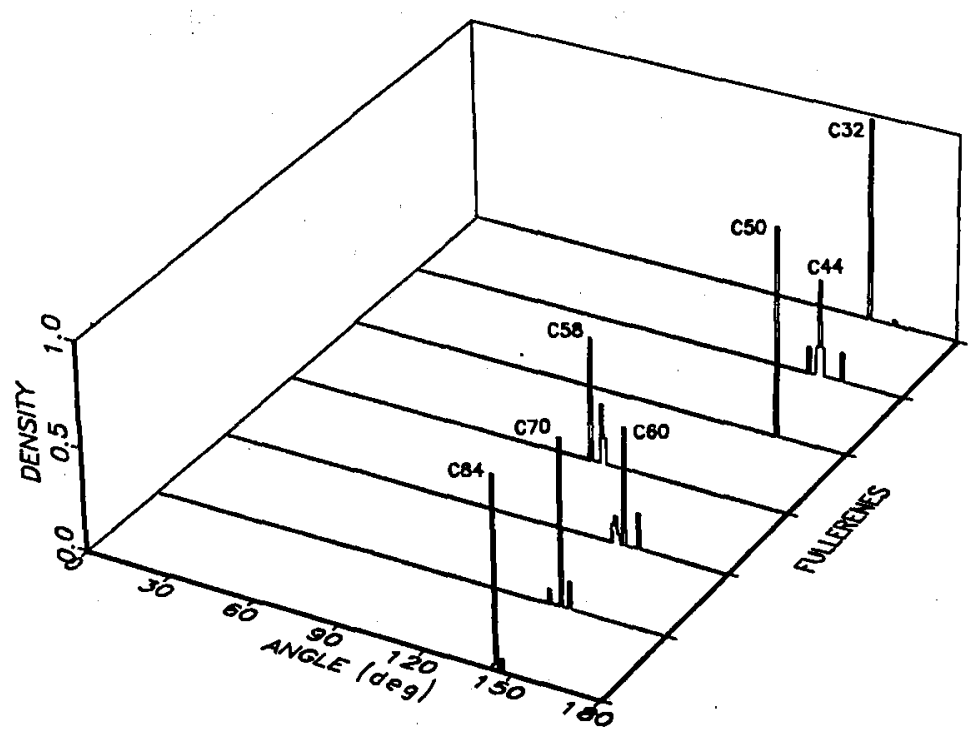

Fig. 1. The probability distribution (density) of the angle of a nearest-neighbour pair of spins on pentagons of the $\mathrm{C}_{N}$ free clusters.

Our results show that $\mathrm{C}_{50}$ is the only exception which has exactly one ground state spin configuration but of non-zero net magnetic moment.

The magnetic ground states of $\mathrm{C}_{N}$ clusters are frustrated because there are pentagons with the negative exchange couplings per bond in the systems. Since the separation of the frustrated pentagons is observed from $\mathrm{C}_{60}$ system, i.e. every pentagon of the molecular structure is surrounded by hexagons only, spin frustrations of respective pentagons in the case interfere with each other only slightly. It results in differences of the magnetic ground state properties between the $\mathrm{C}_{60}$, $\mathrm{C}_{70}, \mathrm{C}_{84}$ clusters and the other ones. It is particularly apparent comparing either the results gathered in Table II and the information about the distributions of angles between neighbouring spins on pentagons of the clusters (Fig. 1). Only in the 
case of the $\mathrm{C}_{60}, \mathrm{C}_{70}$ and $\mathrm{C}_{84}$ clusters the distributions are peaked out around the angle equal to $144^{\circ}$ (i.e. $4 \pi / 5$ ) as should be expected when different pentagons do not further frustrate each other [1]. As seen, $\mathrm{C}_{58}$ cluster performs an intermediate case. Its both local energy minima (valleys) are degenerated, the angles between neighbouring spins on pentagons are quite different than those in any other case, and each ground state spin configuration net magnetic moment is non-zero. The cluster is just the last one with non-separated pentagons. Therefore, it seems that the fullerenes geometrical structure is rebuilt in the case which implies those very special magnetic ground state properties of the cluster.

As compared our results with those presented in [1], we recognized at first the valley structure of the ground states of fullerenes (local minima of the energy were found). We obtained exactly the same lowest energy spin configuration in $\mathrm{C}_{60}$. The configuration corresponds to the situation when there is no frustration beyond that of an elementary pentagon, and spins on a nonpentagon bond are antiparallel. The attained energy is the natural lower bound in the case [1]. Any other frustrations would increase the energy. However, we found that the system has another, close to the first one, local energy minimum (Table I). Moreover, the probabilities of finding the cluster after its rapid cooling in the both minima are comparable (see Table III). Thus you cannot be convinced if one of them is particularly distinguished. Contrariwise to those in [1] our investigations show that $\mathrm{C}_{84}$ cluster does reach the mentioned above lower bound for the energy in this case, as well. Moreover, as seen in Table III, the lowest energy spin arrangement is the most probable and thus distinguished. It seems to be reasonable, because this cluster has the biggest number of hexagons preventing its pentagons from an interference with each other. In our opinion, the presented calculations imply a notion that in principle some magnetic properties of $\mathrm{C}_{60}$ and $\mathrm{C}_{84}$ are similar. Therefore, we do not confirm the conclusion in [1] that $\mathrm{C}_{60}$ cluster is a unique one. Notice that these are the only cases with the zero net magnetic moment in all ground states (Table II). In the case of $\mathrm{C}_{70}$ system our conclusions in general agree with [1]. The cluster does not reach its lower bound energy, although our lowest energy is about $0.1 \%$ lower than that obtained in [1].

The question of a metastable character of the found ground states needs a more refined treatment. Obviously, either quantum spin fluctuations or thermal activation should be taken into account to describe a mechanism for a possible decay of the states. A determination of heights of the energy barriers is the most desired (cf. eg. [2]). Further investigations are in progress.

Two of us (A.K. and M.T.) acknowledge support from grant No. 2 P302 05704 of the Committee for Scientific Research.

\section{References}

[1] D. Coffey, S.A. Trugman, Phys. Rev. Lett. 69, 176 (1992).

[2] S. Ma, Phys. Rev. B 22, 4484 (1980).

[3] L.R. Walker, R.E. Walstedt, Phys. Rev. Lett. 38, 514 (1977); Phys. Rev. B 22, 3816 (1980).

[4] Ch.L. Henley, Ann. Phys. 156, 324 (1984). 